\title{
Surgical approach for borderline "giant" osteoma in the frontal sinus. Clinical case. Literature review
}

\author{
Dzhambazov K., Topalova-Shishmanova A., Traikova N., Anesteva R., Kraeva M. \\ Department of Otorhinolaryngology, Department of Radiology \\ UMHAT "St. George" Plovdiv, Bulgaria \\ Medical University of Plovdiv, Bulgaria
}

\begin{abstract}
Introduction: Osteomas are benign tumors, with slow growth and a long period of asymptomatic development. The paranasal sinuses are often involved, the most affected one being the frontal sinus, followed by the ethmoid, maxillary and sphenoid sinuses. Etiologically, theories of inflammatory, traumatic, genetic genesis should be introduced, but the issue still remains unclear. The diagnosis is very often accidental, when performing radiography or computed tomography (CT). Clinically manifested osteomas with headache, facial deformity, mucocele, intracranial complications are subject to timely surgical intervention. The choice of surgical approach depends on the size of the osteoma and the location, as well as on the professional and technical capabilities given the more specific requirements for the instrument.

Materials and methods: We present a clinical case of a 28-year-old man with complaints of headache in the frontal facial area. An X-ray revealed an osteoma in the area of the left frontal sinus, involving more than $2 / 3$ of its cavity. CT was performed and a discussion of the possibilities of endoscopic endonasal surgery, given the borderline ,giant" osteoma with a diagonal size of about $2.75 \mathrm{~cm}(>3 \mathrm{~cm})$. An endoscopic endonasal approach was applied.

Results: Endoscopic endonasal surgery was performed. The chosen surgical approach was Draf 3 type frontotomy with osteoma extirpation. In early postoperative period- 5 days, there aren't registreted complications. The patient was followed endoscopically in the second week, first month, sixth month. A month later, a control CT scan was performed with data on residuality on the anterior wall of the frontal sinus, without drainage disturbance. Clinically, the patient has no complaints. There are no late postoperative complications.

Conclusion: Symptomatic osteomas of the paranasal sinuses require timely surgery. The choice of surgical approach (open with osteoplastic flap, endonasal or combined) depends on the anatomical location, the size of the tumor, as well as the experience of the surgeon and the technical capabilities of the hospital. Endoscopic endonasal surgery is popular, with highly valued capabilities over time, but in „giant" osteomas it is debatable given the risk of residuality.
\end{abstract}

\section{Introduction}

Osteomas are benign exophytic bone tumors, occurring most often in the area of the skull base and paranasal sinuses, with the most common localization in the frontal and ethmoidal sinuses. Large periosteal osteomas are characteristic of the pelvis, clavicle, and tubular bones. Osteomas are classified as both "true" neoplasms and developmental abnormalities. The onset of osteomas remains asymptomatic for a long time. They are most often diagnosed against the background of another type of pathology or "accidentally"- facial asymmetry, involvement of the orbital area (proptosis, ptosis), severe headache, chronic sinusitis, mucocele. Interesting in the development of osteomas is their association with other diseases, such as Gardner's syndrome (the presence of multiple osteomas in the base of the skull and mandible- odontomas, epidermal and dermoid cysts of the skin. Due to the autosomal dominant inheritance of the syndrome, the patient's family history is of great clinical importance.

Chiu et al. offer classification of osteomas based on three criteria- the size of the osteoma according to the volume of the frontal sinus, the site of attachment and its location relative to the lamina papyracea (sagittal plan).

Grade I- in anteroposterior size in the sagittal plane, the osteoma occupies less than $75 \%$ of the volume of the frontal sinus

Grade II- it occupies more than $75 \%$ of the frontal 
sinus in the sagittal / anteroposterior plane

Grade III- it covers the lateral recess and pass through the lamina papyracea and catches on the upper or anterior wall of the frontal sinus

Grade IV- "massive" osteomas, involving the entire cavity of the frontal sinus

The treatment of the osteomas is surgical. The choice of method- endoscopic endonasal access or external / open- with osteoplastic flap and / or Lynch frontoethmoidectomy is based on the stage of the osteoma. Thus, stage III osteomas are subject to endoscopic endonasal access. Stages three and four are large-volume osteomas and access to them is severely limited.

The aim of the surgical intervention is to functionally restore the physiology of the frontal sinus before the possibility of obliteration with minimally invasive techniques, which is associated with a short postoperative period.

By using the endonasal endoscopic approach, we have the possibility of postoperative follow-up for the presence of residuality or recurrence when the function is restored and there is access to the sinus. Intraoperative complications are associated with a high risk of bleeding from a. ethmoidalis anterior / posterior, skull base involvement, iatrogenic orbital damage (hematomas, n. opticus and n.abducens).

External / open access is significantly more traumatic, with a high risk of dura mater damage, supraorbital and supratrochlear nerve damage, and a risk of subsequent mucocele (Pott's Puffy tumor).

The postoperative period is longer and requires care for surgical wounds, and in the area of the hairy part of the head there is a risk of infections (contact with hair follicles).

\section{Material and methods}

We present a 26-year-old male patient T.B., who is admitted to the Ear, Nose and Throat Clinic at the University Hospital "St. George"- Plovdiv with complaints of severe headache in the left frontal area. He has been repeatedly treated for rhinitis and sinusitis.

Blood test performed-no abnormalities. Appointed $\mathrm{X}$-ray in an outpatient clinic with subsequent computed tomography with data for osteoma in the left frontal sinus (Fig. 1).

After discussion with the patient and his parents, a decision was made to use endoscopic endonasal extirpation of the bone tumor.

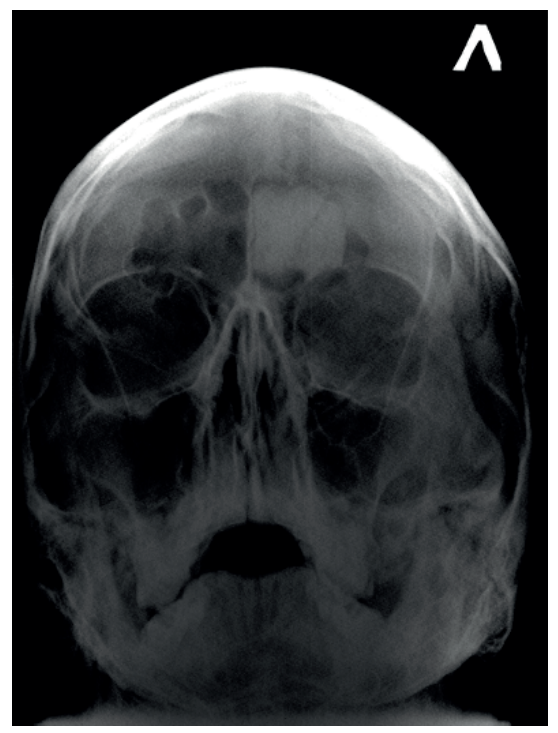

Fig.1. Radiography of the nasal cavities, which diagnoses a bone formation in the left frontal sinus.

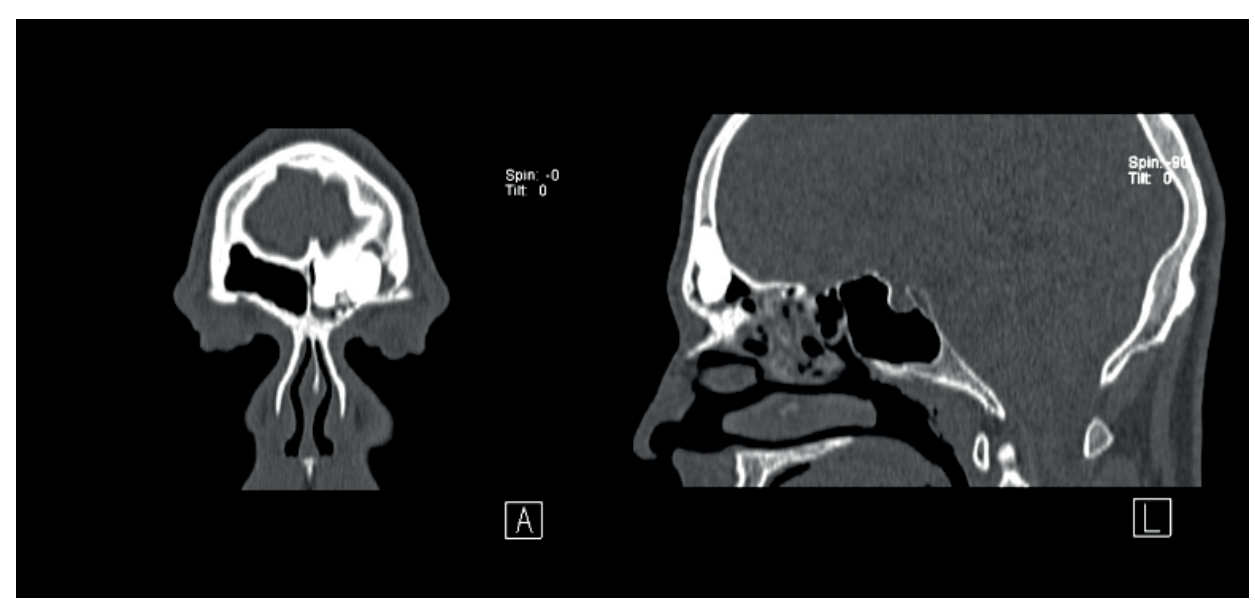

Fig. 2. CT in coronary and sagittal plan with presentation of osteoma. Engaging the three walls of the frontal sinus, occupying over $75 \%$ of the sinus cavity. 


\section{Results}

We applied endonasal endoscopic approach. We performed a resection of the nasal mucosa in the area of the septum in order to present the first olfactory fiber. A frontotomy was performed with a drill and a modo Draf 3. With the help of angular drills, the osteoma was partially extirpated, restoring the functional drainage of the sinus. Due to a defect in the area of the posterior wall of the frontal sinus, but without active cerebrospinal fluid (intact dura), a surgicel was placed. In the early postoperative period, the patient had a periorbital hematoma of the left orbit, but without visual impairment and inflammatory changes in the eye. We did not register late postoperative complications. With clinical improvement over the follow-up period of 3 years. The control CT after 1 month diagnosed residuality, which we left for observation due to the lack of drainage disorders.

\section{Discussion}

When larger than $30 \mathrm{~mm}$ in their maximum dimension, osteomas are termed "giant" or "gigantic". Removing a tumor of this size from the frontal sinus and choosing the most appropriate of all established methods can be a real challenge even for an experienced surgeon. The decision about the correct surgical technique is determined depending on the size, location, spread of the tumor, the available complications, as well as the experience

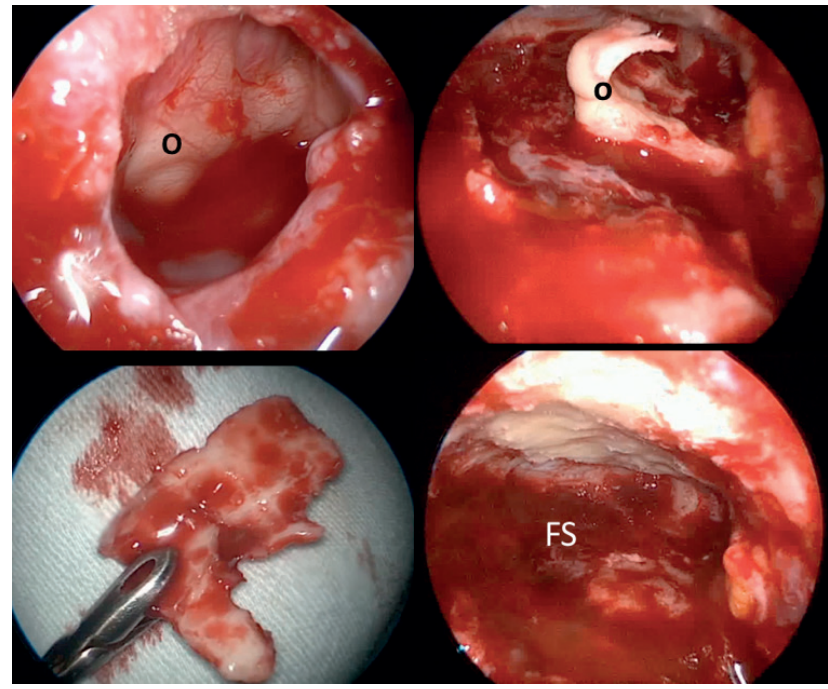

Fig.4. View of endoscopic extirpation of osteoma in frontal sinus. O- osteoma, FS- frontal sinus.

of the surgeon. ${ }^{3}$ Endoscopic access has a number of advantages over other methods. These include less surgical trauma as a result of maintaining healthy tissues, better visualization of those affected, and less intraoperative blood loss. In addition, the lack of postoperative scarring confirms this method as the most aesthetic. According to Gotakis et al. the endoscopic method leads to a significant reduction in postoperative mortality and length of hospital stay. ${ }^{4}$ According to them, complete endoscopic removal of tumors of similar size in many cases may be impossible. One of the major disadvantages of the endoscopic approach is the longer opera-

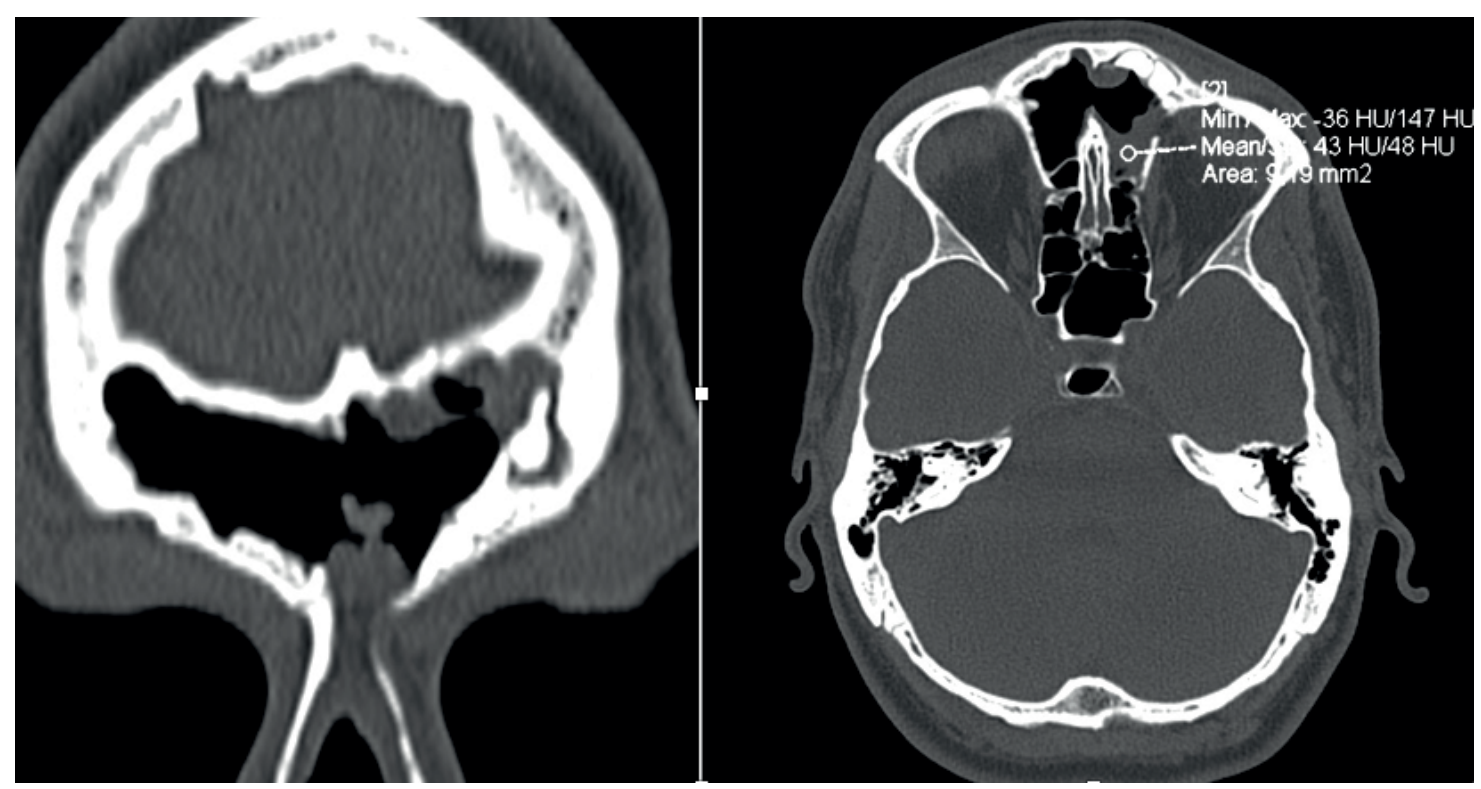

Fig. 3. Coronary and axial plan of CT with data on residuality in the area of the anterior and inferior wall of the frontal sinus. Control endoscopic examinations at 1, 2, 4 weeks, 3 and 6 months clinically confirmed the absence of drainage disorders. 
tive time needed to remove the osteomas. That is why they attach great importance to open surgical techniques, both as a stand-alone treatment and in combination with endoscopic. Arslan et al. indicate that the passage of the osteoma through the lamina papyracea is a clear contraindication to the use of endoscopic access. ${ }^{3}$

When there are no contraindications to its use, endoscopic access in the treatment of "giant" osteoma is applicable, but the risk of residuality must be considered. For this reason a regular follow-up of the patients is appropriate.

On the other hand, the choice of an endoscope is valuable to monitor the frontal recess in combined approaches.

Potential inadequate surgical exposure can also limit its use, especially with far lateral lesion localisation. ${ }^{5}$ Moreover, the resection of healthy normal mucosa with Draf III procedures can lead to frontal recess stenosis. ${ }^{9}$ Modified unilateral osteoplastic flap without obliteration offers good surgical and long-term aesthetic results, especially when a bicoronal incision can be avoided. This approach allows to preserve the mucosa without causing any damage to it, especially with lateral osteomas extending beyond the lamina papyracea. ${ }^{9}$.The surgical approach algorithm referring to Turri-Zanoni et al. shows the clinical steps in choosing a surgical approach and allows us to be as accurate as possible in our assessment.

In our clinical case, the choice of endoscopic endonasal approach according to the algorithm was correct, but the risk of residuality was not avoided. Despite the improved clinical condition, the patient is subject to long-term follow-up.

\section{Conclusion}

Osteomas are benign tumors that are most often found at an advanced stage. The shape and location of the osteoma determine operability, especially in impaired sinus drainage, orbital involvement, and intracranial complications. In our presented case, we demonstrate the positive and negative The choice of surgical method is based on the stage of the osteoma, as well as on the technical capabilities and surgical skills. Endoscopic endonasal surgery is the method of choice given the ability to restore drainage disorders, minimize the risk of intracranial complications and neurological involvement, as well as a significantly shorter postoperative period.

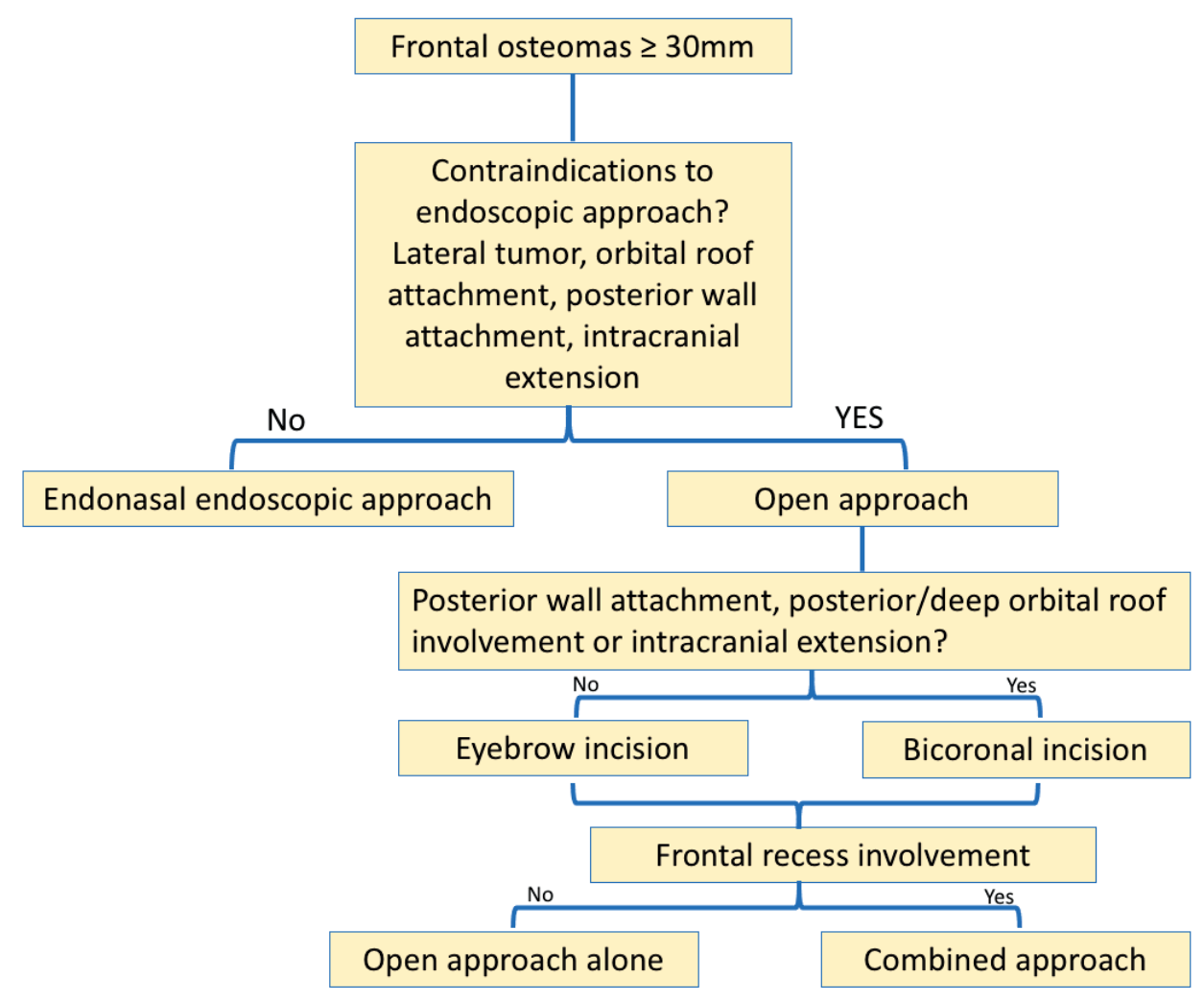

Fig. 5. Surgical approach algorithm for the management of giant frontal sinus osteomas. ${ }^{7}$ 


\section{References}

1. Rangachev Yu., M. Tsekova, T. Popov Tsaritsa Yoanna-ISUL University General Hospital for Active Treatment, Medical University - Sofia, Osteoma of Frontal Sinus. Report of a Clinical Case

2. Osteoma of Frontal Sinus, Divya Gupta, Pankhuri Mittal1, Kavita Goyal1, Ishwar Singh, Department of ENT, Northern Railway Central Hospital, 1Department of ENT, Maulana Azad Medical College and Associated Lok Nayak Hospital, New Delhi, India/ 2018

3. Arslan HH, Tasli H, Cebeci S, Gerek M. The Management of the Paranasal Sinus Osteomas. J Craniofac Surg. 2017 May;28(3):741-745. doi: 10.1097/SCS.0000000000003397. PMID: 28468156.

4. Giotakis E, Sofokleous V, Delides A, Razou A, Pallis G, Karakasi A, Maragoudakis P. Gigantic paranasal sinuses osteomas: clinical features, management considerations, and long-term outcomes. Eur Arch Otorhinolaryngol. 2021 May;278(5):1429-1441. doi: 10.1007/s00405-02006420-x. Epub 2020 Oct 16. PMID: 33064177.

5. Seiberling K, Floreani S, Robinson S, Wormald PJ.Endoscopic management of frontal sinus osteomas revisited. Am J Rhinol Allergy. 2009;23:331-336.

6. Muderris T, Sevil E, Bercin S, Canda B, Kiris M. Giant paranasal sinus osteomas: surgical treatment options. J Craniofac Surg. 2014;25:1287-1291

7. Turri-Zanoni M, Dallan I, Terranova P, et al. Frontoethmoidal and intraorbital osteomas: exploring the limits of the endoscopic approach. Arch Otolaryngol Head Neck Surg. 2012;138(5):498-504.

8. Verillaud B, Le Clerc N, Blancal JP, et al. Mucocele formation after surgical treatment of inverted papilloma of the frontal sinus drainage pathway. Am J Rhinol Allergy 2016;30(5):181-184

9. Meco C, Beton S, Basak H, et al. Periorbital suspension for endonasal endoscopic access to the lateral portion of the frontal anterior skull base. J Neurol Surg B. 2017;78:A157.

10. Rokade A, Sama A.Curr Update on management of frontal sinus osteomas. Opin Otolaryngol Head Neck Surg. 2012 Feb;20(1):40 doi:10.1097/MOO.0b013e32834e9037.PMID: 22249167

11. Georgalas C, Goudakos J, Fokkens WJ. Osteoma of the skullbase and sinuses. Otolaryngol Clin North Am 2011;44:875-890

12. Ledderose GJ, Betz CS, Stelter K, et al. Surgical management of osteomas of the frontal recess and sinus: extending the limits of the endoscopic approach. Eur Arch Otorhinolaryngol 2011;268:525-532

13. Buyuklu F, Akdogan MV, Ozer C, et al. Growth characteristics and clinical manifestations of the paranasal sinus osteomas. Otolaryngol Head Neck Surg 2011;145:319-32

14. Cokkeser Y, Bayarogullari H, Kahraman SS. Our experience with the surgical management of paranasal sinuses osteomas. Eur Arch Otorhinolaryngol 2013;270:123-128

15. Miman MC, Bayindir T, Akarcay M, et al. Endoscopic removal technique of a huge ethmoido-orbital osteoma. J Craniofac Surg 2009;20:1403-1406

16. Bhatki AM, Carrau RL, Snyderman $\mathrm{CH}$, et al. Endonasal surgery of the ventral skull base - endoscopic transcranial surgery. Oral Maxillofacial Surg Clin N Am 2010;22:157-168

17. Selva D, Chen C,Wormald PJ. Frontoethmoidal osteoma: a stereotacticassisted sino-orbital approach. Ophthal Plast Reconstr Surg 2003;19:237-238

Reviewer of the article: Prof. Dr. Rumen Benchev, MD, PhD - University hospital SofiaMed

\section{Correspondence to:}

A.R.Topalova@gmail.com Aleksandrina Topalova-Shishmanova,PhD

ENT Department, UMHAT “St. George” Plovdiv, Medical University of Plovdiv, Bulgaria 